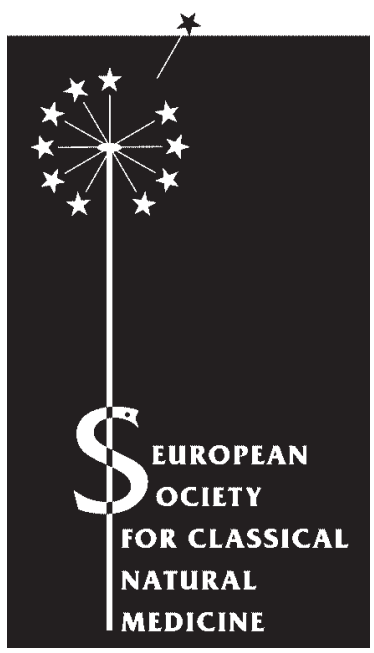

\title{
Gesellschaftsmitteilungen · Society Bulletins
}

Forsch Komplementärmed Klass Naturheilkd 2003;10:108-110

\section{Summer School für klassische Naturheilkunde vom 20. bis 28. September 2003 in Überlingen/Bodensee}

Wie jedes Jahr veranstaltet die ESCNM auch in diesem Jahr eine Summer School für klassische Naturheilkunde vom 20. bis 28. September 2003. Sie richtet sich an Medizinstudierende und will ihnen eine fundierte Einführung in die klassische Naturheilkunde bieten. Unter der Leitung des neuen Vorstandes wird die 6. Summer School den Erfolg der letzten Jahre fortsetzen. Nicht zuletzt durch einen Artikel in der FAZ vom 19.10.02 ist das Interesse an der diesjährigen Summer School bereits sehr gross. Weitere Informationen finden Sie im Internet unter www.summerschool.de.

Die Bodensee-Konferenz für klassische Naturheilkunde wird dieses Jahr in kleinerem Rahmen am Samstag, dem 27.09.03, in Form eines ESCNM Bodensee-Kolloquiums stattfinden. Hier wollen die Mitglieder der ESCNM neue Perspektiven und Projekte entwickeln. Das genaue Programm wird noch bekannt gegeben.

Veranstalter: European Society for Classical Natural Medicine e.V. (ESCNM), mit freundlicher Unterstützung der Erich-Rothenfusser-Stiftung und der Eden-Stiftung

Veranstaltungsort: Aula der Klinik Buchinger am Bodensee, Überlingen

Vorläufiges Programm:

Freitag, 19. September 2003

Ab 19.00 Get together in der Villa Fichtenau

Samstag, 20. September 2003 - Einführung in Naturheilkunde \& Heilfasten

$08.30-09.00 \quad$ Registrierung der TeilnehmerInnen (Aula KB)

$09.00-09.30 \quad$ Begrüssungen

Vorstellungsrunde der TeilnehmerInnen (Aula)

$09.30-09.45$ Organisatorisches, Einteilung in Gruppen (Aula)

Wunderlich

$F 1$ = Fastende, $E 1+E 2$ = Essende; Journalisten; Gruppe, die Studie

ausarbeitet und präsentiert; Service; Bodenseekonferenz

09.45 - $11.00 \quad$ Die Basis der Naturheilkunde: Wie kann ich die eigenen

Selbstheilungskräfte steigern? (Aula) Wilhelmi

$11.15-12.00 \quad$ Heilfasten nach Buchinger: Ein klassisches Naturheilverfahren (Aula)

Wilhelmi und

MitarbeiterInnen

der KB

$12.05-13.00$

Gruppe E 1: Praxis Ernährungstherapie: Zubereitung des Mittagessens (Pavillon)

Hohler

$12.15-12.45$

Gruppe $E 2+$ Fastengruppe ( $F$ 1): Praktische Einführung in das Fasten (Aula)

Lüttich

$12.50-1315$

F 1: Entlastungsmahlzeit (Speisesaal, Haupt-Haus)

Lüttich

$16.00-17.45$

Forschung: Vorstellung der didaktischen Studie (Aula)

$\mathrm{ZNF}$

$19.30-21.00 \quad$ Vortrag: «89 verschiedene Schlafstörungen und ebenso viele naturheilkundliche Behandlungsmassnahmen»

Hecht

\section{KARGER}

(C) 2003 S. Karger GmbH, Freiburg 
Sonntag, 21. September 2003 - Chronobiologie und Schlaf. Phytotherapie

09.00-10.30 Chronopsychobiologie: Chronomedizin, Psychobiologie des Schlafes, Hecht

$\begin{array}{ll}11.10-12.00 & \text { Praxisseminar-Minischlaf (Aula) } \\ 12.05-13.00 & \text { E 1: Praxis Nahrungszubereitung (Pavillon) }\end{array}$

Hecht

Hohler

12.05-12.45 E $2+F 1$ : Sprechstunde Psychologie des Fastens (Aula)

13.45 - 14.15 Datenerfassung Studie (Doktorhaus, Bibliothek)

Wilhelmi

14.15-16.15 Phytotherapie: Kräuterwanderung im Hödinger Tobel,

Pflanzen wahrnehmen \& kennenlernen - ihre Anwendung in der

homöopathischen und anthroposophischen Medizin

$16.30-18.00 \quad$ Praxsisseminar - Kräutertees und Präparate, Wickel, Auflagen

NN

Schubert

Schubert

Montag, 22. September 2003 - Ordnungs-/Bewegungstherapie

$09.00-10.00 \quad$ Ordnungstherapie (naturheilkundliche Gesundheitspädagogik) (Aula)

10.15 - 11.00 Bewegungstherapie: Theorie - Art, Intensität und Dauer der Bewegung nach Indikation und individueller Ausgangslage

Naturheilkundliche Betrachtungsweise der Bewegungstherapie:

-Psychohygienische Wirkungen der Bewegung

Wunderlich

-Aufgaben und Möglichkeiten der Ärzte (Aula)

11.15-12.00 Bewegungstherapie: Theorie - Ausdauertraining (Aula)

12.05 - 13.00 2: Praxis Ernährungstherapie: Zubereitung des Mittagessens (Pavillon)

12.05-12.45 E $1+F 1$ : Sprechstunde Darmhygiene (Aula)

$14.30-17.20 \quad$ Praxis der Bewegungstherapie in zwei Gruppen (vor Aula / bei Regen Gymn. Halle)

$17.30-18.00$

Bewegung (Theorie) - Gruppenarbeit/Gruppendynamik (Aula)

19.30

Vortrag Psychologie (Aula)

$\mathrm{NN}$

$\mathrm{NN}$

Hohler

Wilhelmi

NN

NN

NN

\section{Dienstag, 23. September 2003 - Phytotherapie}

$09.00-11.30 \quad$ Phytotherapie: Definitionen, Begriffe, Erfahrungshintergrund, Evidenz bevorzugt an Beispielen Johanniskraut und Pestwurz, Rahmenbedingungen für Zulassung und Verordnung, Phytotherapeutische Kombinationspräparate (Aula)
$11.30-12.00$
$12.05-13.00$
$12.05-12.45$
$14.00-16.00$

Besichtigung des Kräutergartens der Klinik Buchinger

E 2: Praxis Nahrungszubereitung (Pavillon)

E 1 F 1: Sprechstunde Befindlichkeit und Krisen (Aula)

Phytotherapie: Ätherische Öle als vielschichtige Phytotherapeutika,

Teezubereitungen als einfache Phytotherapeutika und Hausmittel bevorzugt an den Beispielen Pfefferminze und Baldrian, Teemischungen

Diskussionen und Fragen (Aula)
16.30-18.30 Kunstbetrachtung: Seminar, Der Anfang aller Kunstbetrachtung ist ein Stuhl (Aula)

$19.30-21.00 \quad$ Vortrag: Der Anfang aller Kunstbetrachtung ist ein Stuhl (Aula)

Saller

Saller

Hohler

Wilhelmi

Saller

Stoll

Stoll

Mittwoch, 24. September 2003 - Physio- und Hydrotherapie/Balneologie/Moortherapie

9.20 Tagesausflug nach Bad Waldsee; Ankunft in der Waldsee-Therme

$9.30-10.00 \quad$ Begrüssung durch Herrn Prof. Rudolf Forcher, Bürgermeister und Kurdirektor Bad Waldsee, Vorsitzender des Baden-Württembergischen Heilbäderverbandes

10.00 - $11.15 \quad$ Rheumatherapie im Spannungsfeld zwischen Schulmedizin und Naturheilverfahren

$11.30-12.15$

Balneologie in der Gynäkologie

$12.15-13.00$
Forcher

Maier

Jentsch

Jentsch/Maier 
$14.00-15.30 \quad$ Selbsterfahrung mit Moorbad, Moorpackung, Wechselguss, Heusack,

Kamm $\mathrm{CO}_{2}$-Trockenanwendung

16.00 - $17.30 \quad$ Demonstration unterschiedlicher Massageformen:

Gerstlauer

klassische detonisierende Massage, Bindegewebsmassage, Lymphdrainagen und

Fussreflexzonenmassagen

17.40 - $19.00 \quad$ Rückfahrt nach Überlingen

19.30 Vortrag: Naturheilkundliche Ernährungstherapie

Prophylaktische und therapeutische Wirkungen der Ernährung zwischen

Vollwertigkeit und Verträglichkeit

Donnerstag, 25. September 2003 - Ernährung/Forschung

09.00 - 09.45 Ernährungstherapie: Die drei Dimensionen der Ernährung: gesundheitliche, ökologische und soziale (Aula)

$09.45-10.30$ Wo «Bio» draufsteht, ist auch Bio drin?! Bio-Lebensmittel als Element einer gesunden und nachhaltigen Ernährung (Aula)

10.45-12.00 Naturheilkunde: Ausleitende Verfahren: Schröpfen, Blutegel (Aula)

12.05-13.00 E 1: Praxis Ernährung (Pavillon)

12.05-12.45 E2 $+F 1$ : Theorie Ernährungstherapie - Empfehlungen für die

Lebensmittelauswahl, Fragen und Diskussionen (Aula)

13.45 - $14.30 \quad$ Studiengruppe: Datenerfassung Studie (Doktorhaus, Bibliothek)

$14.45-16.15 \quad$ Diagnostik in der Naturheilkunde

v. Koerber

v. Koerber

Kraft

Hohler

v. Koerber

Kraft

Jena

v. Koerber

Hohler

v. Koerber

ZNF
20.45

Therapeutische Wirkungen der Musik

Ernährungstherapie: Theorie - Einfluss des Frühstücks auf Glykämie und Leistungsbereitschaft (Aula)

$12.00-12.50$

E 2: Raumgestaltung Service (Pavillon)

12.00-12.40 E 1: Theorie Ernährungstherapie - Empfehlungen für die Lebensmittelauswahl, Fragen und Diskussionen (Aula)

12.15 Pressekonferenz (Salon im Haus Belgrano, KB)

$14.00-18.00 \quad$ Ausflug nach Allensbach zum Institut für Demoskopie

bzw.

$14.00-18.00$

Vorstellung der Daten zu Themen der Naturheilkunde

Forschung in der Naturheilkunde

z.B. allgemeine Grundlagen der «evidence-based medicine»,

Studiendesign usw. (Aula)

Präsentation der Stiftungen, die die Summer School unterstützen

Samstag, 27. September 2003 - ESCNM Bodensee-Kolloquium für klassische Naturheilkunde

$09.00-16.00 \quad$ ESCNM Bodensee-Kolloquium:

Präsentation der didaktischen Studie der Summer School

Referate von ESCNM-Mitgliedern

16.00 - 18.00 Vorstandssitzung mit Workshop zur Entwicklung der ESCNM

\section{Sonntag, 28. September 2003}

$08.00-8.30 \quad$ Frühstück in der Klinik Buchinger, Abfahrt 